\title{
Follow-up of Thalidomide treatment in patients with Hereditary Haemorrhagic Telangiectasia*
}

\author{
A.E. Hosman', C.J.J. Westermann², R. Snijder², F. Disch³, C.L. Mummery4, \\ J.J. Mager ${ }^{2}$
}

Rhinology 53: 340-344, 2015

DOl:10.4193/Rhino14.289

*Received for publication:

November 27, 2014

Accepted: March 28, 2015

2 Department of Pulmonary Medicine, St Antonius Hospital, Nieuwegein, the Netherlands

${ }^{3}$ Department of ENT Medicine, St Antonius Hospital, Nieuwegein, the Netherlands

${ }^{4}$ Department of Anatomy and Embryology, Leiden University Medical Centre, the Netherlands

\begin{abstract}
Background: Patients with a hereditary vascular disorder called Rendu-Osler-Weber syndrome (Hereditary Haemorrhagic Telangiectasia, HHT) haemorrhage easily due to weak-walled vessels. Haemorrhage in lungs or brain can be fatal but patients suffer most from chronic and prolonged nosebleeds (epistaxis), the frequency and intensity of which increases with age. Several years ago, it was discovered serendipitously that the drug Thalidomide had beneficial effects on the disease symptoms in several of a small group of HHT patients: epistaxis and the incidence of anaemia were reduced and patients required fewer blood transfusions. In addition, they reported a better quality of life. However, Thalidomide has significant negative side effects, including neuropathy and fatigue.
\end{abstract}

Methods: We followed up all HHT patients in the Netherlands who had been taking Thalidomide at the time the original study was completed to find out (i) how many had continued taking Thalidomide and for how long (ii) the nature and severity of any side-effects and (iii) whether side-effects had influenced their decision to continue taking Thalidomide.

Results: Only a minority of patients had continued taking the drug despite its beneficial effects on their symptoms and that the side effects were the primary reason to stop.

Conclusion: Despite symptom reduction, alternative treatments are still necessary for epistaxis in HHT patients and a large-scale clinical trial is not justified although incidental use in the most severely affected patients can be considered.

Key words: Telangiectasia, Hereditary Hemorrhagic, Thalidomide/adverse effects, Thalidomide/therapeutic use, epistaxis

\section{Introduction}

Thalidomide, a drug also known as Softenon, is by many associated with its notorious side-effects on fetal development in humans in the 1960s. Babies born to mothers taking Thalidomide during early pregnancy for nausea often failed to develop limbs and had multiple other severe birth defects. Thalidomide was discredited but has more recently been reintroduced and is now successfully used in the treatment of multiple myeloma and other afflictions ${ }^{(1)}$. It is now also being investigated and used in the treatment of symptoms associated with Hereditary Haemorrhagic Telangiectasia (HHT, also known as Rendu-OslerWeber syndrome). This is an autosomal dominant disease is characterised by multi-systemic vascular lesions, afflicting about
1 in 5000 people ${ }^{(2-4)}$. It is caused by mutations in receptors of the transforming growth factor $\beta$ family, most notably endoglin and activin receptor like kinase type 1 (ALK1), which are expressed on endothelial cells of blood vessels ${ }^{(5)}$. Symptoms include telangiectasia, blood vessel abnormalities typically evident around lips, oral mucosa and fingertips. Gastrointestinal and nasal bleeding often lead to chronic iron deficiency, anaemia, and complications due to liver-, lung- and brain arteriovenous malformations ${ }^{(6)}$. For patients, anaemia due to gastrointestinal bleeding and social limitations due to nasal bleeding have the most effect on the quality of daily life. Animal and patient studies have shown Thalidomide has a positive effect on HHT related symptoms ${ }^{(7,8,13-18)}$. However, Thalidomide is also known 
to coincide with severe side-effects including neuropathy, severe skin reactions, angina and dyspnoea, oedema, drowsiness, general malaise and tremor. It has also been reported to cause deep vein thrombosis in one patient using Thalidomide to control nosebleeds secondary to HHT. The mechanism of action of Thalidomide in patients with $\mathrm{HHT}$ has been extensively discussed in Lebrin et al. ${ }^{(7)}$. In short, Thalidomide promotes vessel maturation and enhances the coverage of smooth muscle cells around vessels, making vessels more stable and lowering nosebleed frequency in HHT subjects. The cohort of patients included in the first study ${ }^{(7)}$ was relatively small and the patients served as their own (historical) controls. These patients and others who were prescribed Thalidomide later, were, however, never systematically followed up to evaluate the long-term outcome, severity of side effects and drug compliance. The current study was designed for this purpose with the view to considering a larger scale, double blinded trial.

\section{Materials and methods}

\section{Patient cohort}

The St. Antonius Hospital is the only HHT-center in the Netherlands and most patients in the Netherlands are known to the hospital or are treated by the group of specialists authoring this paper. In general, HHT is diagnosed clinically using the Curacao criteria ${ }^{(5)}$ in which patients are scored for evidence of nosebleeds, mucocutaneous telangiectasia, visceral involvement (most commonly gastrointestinal telangiectasia, or AVMs at specified sites), and a family history of HHT. Severely affected patients are often referred to the St Antonius Hospital for treatment of nosebleeds if the frequency of blood transfusions, degree of anaemia is excessive. The patients who have also often had nasal cauterization without prolonged success are those that have been offered Thalidomide prior to the final option of the Saunder's operation. This means that the patients included in this study are a subset of all patients in the Netherlands but are among the most severely affected subset. In 2014, there were $1283 \mathrm{HHT}$ patients on record in the St. Antonius Hospital, of whom less than $1 \%$ were treated with Thalidomide. All patients who received Thalidomide between 2009 and 2014 made up the cohort for this study as no patients in the Netherlands are treated with Thalidomide elsewhere than the St Antonius Hospital.

\section{Questionnaire}

To capture information on patient experience with Thalidomide, a paper questionnaire was used to collect data. The data was returned anonymously and was analysed using Excel and Graph Pad Prism 5 (GraphPad Inc, US). The questionnaire was composed of general health questions, symptoms of HHT before taking Thalidomide, after 3 months and currently or just before ceasing Thalidomide use and personal experience. In an attempt to assess the severity of symptoms objectively, HHT patients provided information on the number of nosebleeds per week, the mean duration of each nosebleed and the number of blood transfusions needed due to severe anemia.

The questionnaire was a retrospective collection of data but 4 patients had already been included in the initial study (shortterm follow-up) published in Nature Medicine ${ }^{(7)}$. In that study, patients kept a detailed diary of nosebleeds (frequency and severity) in the three months before and after treatment. This is

Table 1. Severity of epistaxis.

\begin{tabular}{|c|c|c|c|c|c|c|c|c|c|c|c|c|}
\hline \multirow[t]{2}{*}{ No } & \multicolumn{4}{|c|}{ Before Thalidomide use } & \multicolumn{4}{|c|}{ After 3 months of Thalidomide } & \multicolumn{4}{|c|}{ Current situation or just before stopping } \\
\hline & severity & $n / w$ & duration & transfusion & severity & $\mathrm{n} / \mathrm{w}$ & duration & transfusion & severity & $\mathrm{n} / \mathrm{w}$ & duration & transfusion \\
\hline 1 & $* * *$ & & & & $* *$ & & & & & & & \\
\hline 2 & $* * *$ & 15 & 30 & & $* *$ & 7 & 20 & 0 & * & & & \\
\hline 3 & $* * * *$ & & $30-60$ & 1 & ** & 1 & $15-30$ & 0 & ** & 0 & 0 & 0 \\
\hline 4 & $* * * *$ & 29 & 10 & 3 & $* *$ & 2 & 5 & 0 & * & 2 & 5 & 0 \\
\hline 5 & $* * * *$ & 50 & $15-90$ & 12 & $* *$ & 13 & 15 & 4 & * & 2 & 5 & 4 \\
\hline 6 & $* * *$ & 210 & $1-5$ & 2 & $* *$ & 10 & $1-5$ & 2 & & 2 & $1-5$ & 0 \\
\hline 7 & $* * * *$ & 45 & $5-45$ & 10 & $* * *$ & 20 & 15 & 6 & & & & 0 \\
\hline 8 & $* * * *$ & 4 & $3-60$ & 2 & $* *$ & 2 & 10 & 0 & * & 1 & $1-2$ & 0 \\
\hline 9 & $* * * *$ & 1 & $10-60$ & & $* * *$ & & 50 & & & & & \\
\hline 10 & $* * * *$ & 7 & & 0 & $* *$ & 5 & & 0 & & & & \\
\hline 11 & $* * *$ & 7 & & & & & & & & & & \\
\hline 12 & $* * * *$ & 40 & $5-30$ & 0 & $* *$ & 15 & 5 & 0 & $* *$ & 15 & 5 & 0 \\
\hline mean & & 40.8 & 25 & 3.75 & & 7.75 & 18 & 1.33 & & 5.67 & 3.8 & 0.57 \\
\hline
\end{tabular}

Severity score: * mild, ${ }^{* *}$ medium, ${ }^{* * *}$ severe, ${ }^{* * * *}$ extreme. N/w: number of epistaxis per week. Duration of epistaxis is measured in minutes. "Transfusions" indicates the number of blood transfusions received in that period. 


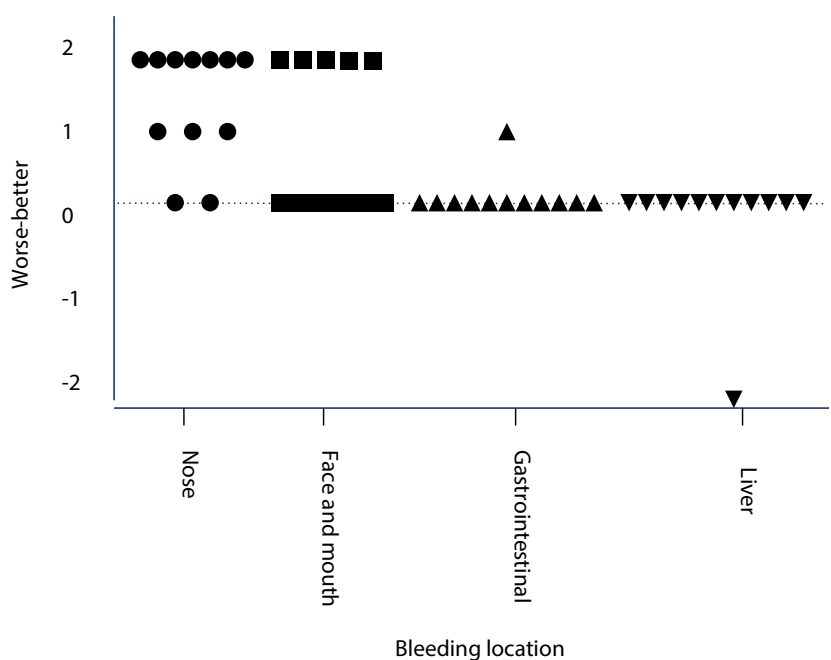

Figure 1. Difference in bleeding in nose, facial and oral area, gastrointestinal and liver haemorrhage using Thalidomide scored -2 "much worse", -1 "worse", 0 " no difference", 1 " better", 2 "much better".

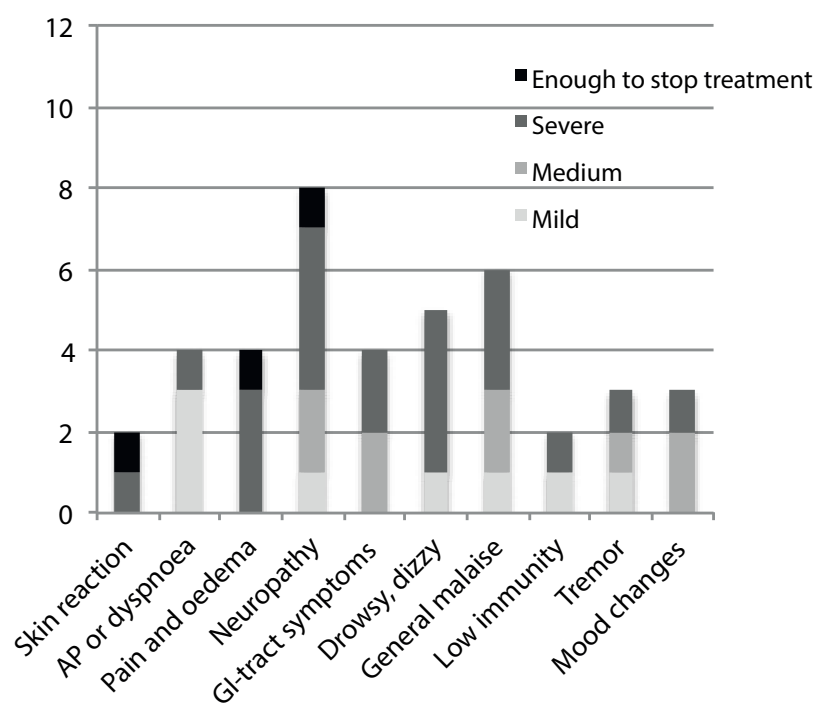

Figure 2. Side- effects experienced by patients. Side-effects are shown on the $x$-axis, the number of patients on $y$-axis. Degree of severity is color-coded. routine procedure for this subgroup of patients that are particularly severely affected. These patients included in the present study are therefore used to monitoring their own symptom severity. Furthermore, even though most of the data is subjective, the number of blood transfusions received in a specific period is objective.

\section{Ethical approval}

The questionnaire was approved for use in this study by the Medical Ethical Committee of the St Antonius Hospital, Nieuwegein, the Netherlands.

\section{Results}

Nineteen patients met the criteria from records to be included. However, 4 patients had died from non-HHT related causes, 1 patient declined to participate and 2 did not respond, leaving 12 participants. The cohort was made up out of 6 males and $6 \mathrm{fe}-$ males with a median age of 69.5 years [IQR $60.0-80.5$ ]. Subjects had been treated daily with 50 to $100 \mathrm{mg}$ of Thalidomide orally. The most common types of treatment patients had undergone previously are reported: 11 of 12 patients used iron tablets for iron deficiency anaemia, 8 had undergone argon laser treatment, 7 had used Sofradex, 6 had been subjected to cauterisation, 4 had undergone embolization, and 3 had nasal epithelium transplantation (Saunder's operation). Thalidomide was used for a median time of 7 months [IQR 3.0 - 23.3]. Reported influence on frequency and severity of nosebleeds is shown in Table 1. The number of blood transfusions each patient underwent in the study period is also given in Table 1 . This is an objective measure of disease severity.
In the questionnaire, differences in symptoms during Thalidomide use could be scored by the patients as: much worse, worse, no difference, better and much better (Figure 1). Three patients rated their nosebleeds "better" during Thalidomide use, and 7 patients thought their nosebleeds were "much better". Five patients rated oral bleedings or bleeding located in the facial area as much better. Remaining patients did not notice any difference at these sites. One patient reported having had a liver haemorrhage even though this is an exceptionally rare complication. However, according to our patient records, none of the participating patients has ever had a liver haemorrhage raising doubt on whether this patient understood the question.

Four patients were still using Thalidomide after respectively $3,27,30$ and 50 months. One patient stopped because of the lack of effect on their symptoms and the remaining 7 patients discontinued use because of side-effects (Figure 2). When asked if the advantages of Thalidomide weighed up against the disadvantages, 5 participants reported they agreed, 3 returned neutral answers and 4 disagreed. Nine out of 12 patients would recommend trying Thalidomide to other HHT patients.

\section{Discussion}

The majority of HHT patients included here had tried an array of therapies for reducing nosebleeds by the time they were considered for Thalidomide treatment. These included compression techniques, bilateral embolization, surgical arterial ligature, laser therapy, sclerosing agents, electrocautery, septodermoplasty, adjuvant medical treatments including estrogens, progeste- 
rone and antifibrinolytic agents. Most relieve symptoms only temporarily so that there is a significant unmet medical need for a more stable, less invasive alternatives ${ }^{(8,9)}$.

Several reports have described beneficial effects of Thalidomide on some of the symptoms of HHT: nosebleeds were reduced in some cases and as a direct or indirect result secondary symptoms, caused by blood loss, were also reduced ${ }^{(7)}$. Similar results were described by Balduini et al. ${ }^{(18)}$ in a preliminary study of 11 patients. Reduced nosebleed frequency was observed in 5 within a few weeks of initiating treatment. In addition, in vivo studies in mutant mice heterozygous for the Endoglin gene and in HHT patients as well in vitro studies, have indicated a mechanism through which Thalidomide could act in promoting vessel stability: up regulation of the expression of platelet derived growth factor- $\beta$ (PDGF- $\beta$ ) by endothelial cells results in enhanced recruitment of pericytes expressing PDGF receptors to the vessel wall. The pericytes subsequently differentiate to smooth muscle cells as a result of this interaction with endothelial cells and improved vessel maturation occurs. In addition, Thalidomide increases the number of smooth muscle cells in mice ${ }^{(7)}$ and in the nasal epithelium of HHT patients. Furthermore, anti inflammatory and immunosuppressive properties of Thalidomide have been hypothesized as preventing de novo pulmonary arteriovenous malformation development ${ }^{(10)}$. This would bode well for optimism in the use of Thalidomide to treat HHT and, indeed, the vast majority of participants in this study reported an improvement in their symptoms. However, side-effects seemed to be a limiting factor for use in practice. Neuropathy was reported in 8 out of 12 patients of whom more than half reported the neuropathy to be severe. Although this study group in total is very limited in size, severe side effects have been reported in patients with multiple myeloma treated with Lenalidomide by Katodritou et al. ${ }^{(11)}$. Adverse events were reported in $68.9 \%$ of patients (myelosuppression in $49.4 \%$ ) and $12.7 \%$ of patients needed hospitalization. Peripheral neuropathy was reported in $2.5 \%$ of patients and deep vein thrombosis in $5.7 \%$. Almost $39 \%$ of patients discontinued the treatment completely due to side-effects. Overall, 34 cases in ten studies of HHT patients treated with Thalidomide and Bevacizumab have been reported. Follow-up varies from 1 to 60 months (mean 13.5 months) $(6,12-20)$. Although the majority of cases reported Thalidomide as being a successful treatment with reduced blood transfusions and epistaxis, adverse reactions were often underreported or not mentioned. One patient developed deep vein thrombosis (16), although it is questionable whether the Thalidomide was directly responsible. Although no other major adverse events are reported, some patients discontinued the treatment without further documentation on motives.

\section{Conclusion}

Overall, Thalidomide appears to be beneficial for treating HHT even though it is effectively one of many Thalidomide side effects ("off target use"). Yet the severity of the side-effects would likely be a disincentive to begin such a trial where the chance that patients would continue using the drug long term are somewhat limited. Nevertheless, in particularly severe cases where other treatments have been exhausted, there may be justification for incidental use. Whilst most insurance companies or health systems would not cover the cost of a drug under these circumstances, the costs of Thalidomide are low and for the patients in this study, the costs were not prohibitive enough to opt out of the study.

\section{Acknowledgements}

The authors are grateful to the patients at the Netherlands HHT Expertise Centre for their willingness to to participate in this study. In addition, we thank Lucia Mijs, Daniëlle Barendregt and Margré Kooman of the CGS Prins Maurits School in Middelharnis for their initiation and enthusiastic participation in this project and their contribution to the questionnaire and its analysis. HHT studies at the St Antonius Hospital and LUMC are supported by the SWORO Foundation for HHT patients.

\section{Author contributions}

$\mathrm{AEH}$ designed the questionnaire, collected data, analysed data, performed a literature search and wrote the initial article drafts. CJJW contributed designing the questionnaire and writing the article draft. RS and FD contributed in writing the final manuscript. JJM managed patient recruitment and with CLM, finalized the manuscript.

\section{Conflict of interest}

We certify absence of conflict of interest with any financial organization regarding the material discussed in the manuscript.

\section{References}

1. Eriksson T, Björkman S, Höglund P. Clinical pharmacology of thalidomide. Eur J Clin Pharmacol. 2001; 57: 365-376.

2. Kjeldsen AD, Vase P, Green A. Hereditary haemorrhagic telangiectasia: populationbased study of prevalence and mortality in Danish patients. J Intern Med. 1999, 245

\section{1-39.}

3. Dakeishi M, Shioya T, Wada Y, et al. Genetic epidemiology of hereditary hemorrhagic telangiectasia in a local community in the northern part of Japan. Hum Mut. 2002; 19: 140-148.

4. Plauchu $H$, de Chadarévian JP, Bideau A, Robert JM. Age-related clinical profile of hereditary hemorrhagic telangiectasia in an epidemiologically recruited population. Am J Med Genet. 1989; 32: 291-297.

5. Shovlin CL. Hereditary haemorrhagic telangiectasia: pathophysiology, diagnosis and treatment. Blood Rev. 2010; 24: 203-219.

6. Govani FS, Shovlin CL. Hereditary haemorrhagic telangiectasia: a clinical and scientific 
review. Eur J Hum Genet. 2009, 17: 860-871.

7. Lebrin F, Srun S, Raymond K, et al Thalidomide stimulates vessel maturation and reduces epistaxis in individuals with hereditary hemorrhagic telangiectasia. Nat Med. 2010; 16: 420-428.

8. Franchini M, Frattini F, Crestani S, Bonfanti C. Novel treatments for epistaxis in hereditary hemorrhagic telangiectasia: a systematic review of the clinical experience with thalidomide. J Thromb Thrombolysis. 2013; 36: 355-357.

9. Silva BM, Hosman AE, Devlin HL, Shovlin CL. Lifestyle and dietary influences on nosebleed severity in hereditary hemorrhagic telangiectasia. Laryngoscope. 2013; 123 1092-1099.

10. Lacout A, Marcy PY, El Hajjam M, Lacombe P. Pulmonary arteriovenous malformations etiologies in HHT patients and potential utility of thalidomide. Med Hypotheses. 2013; 80: 587-588.

11. Katodritou E, Vadikolia C, Lalagianni C, et al: "Real-world" data on the efficacy and safety of lenalidomide and dexamethasone in patients with relapsed/refractory multiple myeloma who were treated according to the standard clinical practice: a study of the Greek Myeloma Study Group. Ann Hematol. 2014; 93: 129-139.

12. Kursting R. Using Thalidomide in a patient with epithelioid leiomyosarcoma and Osler-
Weber-Rendu disease. Oncology (Wilston park) 2002; 16: 21-24.

13. Kersemeakers MAH, Westermann CJJ, Mager JJ, De Weerdt O, Disch FMJ, Snijder RJ. Thalidomide for the treatment of epistaxis in hereditary hemorrhagic telangiectasia. Hematol Meet Rep. 2007; 1: 22-23.

14. Buscarini E, Manfredi G, Gazzaniga P, et al. on behalf of HHT-NET Thalidomide for the treatment of chronic severe bleeding in hereditary hemorrhagic telangiectasia. Hematol Meet Rep. 2009; 3: 20-21.

15. Gossage JR, Chamberlain SM, Sridhar S, Kumar A. An interim report of thalidomide treatment of recurrent angioectasia related gastrointestinal bleeding. Hematol Meet Rep. 2009; 3: 21

16. Penaloza A, Vekemans MC, Lambert $C$, Hermans C. Deep vein thrombosis induced by thalidomide to control epistaxis secondary to hereditary hemorrhagic telangiectasia. Blood Coagul Fibrinolysis. 2011; 22: 616-618.

17. Alam MA, Sami S, Babu S. Successful treatment of bleeding gastro-intestinal angiodysplasia in hereditary haemorrhagic telangiectasia with thalidomide BMJ Case Rep. 2011; 10:1136.

18. Balduini $C L$, Pallega $F$, Bellistri $F$, et al. Efficacy of thalidomide in treatment of severe recurrent epistaxis in hereditary hemorrhagic telangiectasia (HHT): ongo- ing results of a prospective study. Blood Transfus. 2012; 10: s36.

19. Chavan A, Schumann-Binarsch S, Luthe L, et al. Systemic therapy with bevacizumab in patients with hereditary hemorrhagic telangiectasia (HHT). Vasa. 2013; 42: 106-110.

20. Chen $\mathrm{CH}, \mathrm{Hsu} \mathrm{HH}, \mathrm{Hu} \mathrm{RH}$, et al. Long-term therapy with thalidomide in hereditary hemorrhagic telangiectasia: case report and literature review. J Clin Pharmacol. 2012; 52: 1436-1440.

\section{JJ Mager}

St Antonius Hospital

Department of Pulmonary Medicine

and Tuberculosis

Postbox 2500

3430 EM Nieuwegein

the Netherlands

Tel: +31-88-320 1425

Fax: +31-88-320 1449

E-mail:

jjmager@antoniusziekenhuis.nl 